\title{
Family-based suppressive intermittent therapy of hyperendemic trachoma with topical oxytetracycline or oral doxycycline
}

\author{
S. DAROUGAR, ${ }^{1}$ B. R. JONES, ${ }^{1}$ N. VISWALINGAM, ${ }^{1}$ R. H. POIRIER, ${ }^{2}$ \\ J. ALLAMI, ${ }^{3}$ A. HOUSHMAND, ${ }^{3}$ M. A. FARAHMANDIAN, ${ }^{4}$ AND J. A. GIBSON ${ }^{1}$ \\ From the ${ }^{1}$ Department of Clinical Ophthalmology, Institute of Ophthalmology, Judd Street, \\ London WC1H 9QS, the ${ }^{2}$ Department of Ophthalmology, University Texas Health Science Center, \\ San Antonio, USA, the ${ }^{3}$ School of Public Health, Teheran University, Iran, and the \\ ${ }^{4}$ Department of Health and Welfare, Bandar-Abbas, Iran
}

SUMMARY A controlled double-blind stratified trial was carried out in a village in Southern Iran to assess the efficacy of family-based intermittent therapy of hyperendemic trachoma with topical oxytetracycline oily suspension twice daily for 7 days each month, or oral doxycycline $5 \mathrm{mg}$ per kilogram of body weight once a month, in comparison with a control group which received vitamin pills once a month. In addition all other members of the selected children's families were also treated with the same regimen of therapy. The treatment was given for a period of 1 year by 3 field technicians, each responsible for one regimen of therapy. Examining the whole conjunctiva 4 months after the start of therapy, we observed no marked difference in the cure rate or the number of patients with moderate to severe trachoma between the groups treated with antibiotics and the control group. When treatment was continued for 12 months, a marked decrease in the prevalence of trachoma and in the grades of intensity of inflammatory responses as well as the positivity rate for Chlamydia trachomatis was observed in the groups treated with the topical oxytetracycline or oral doxycycline compared with the control group. While there was no marked difference between the efficacy of these 2 regimens of mass chemotherapy, the monthly intermittent therapy with a single dose of doxycycline offers the advantage of being more practical and less expensive for mass control of trachoma by requiring approximately one-tenth of the staff, transport, and other facilities required for the intermittent topical therapy with tetracycline eye ointment.

Intensive topical therapy with tetracycline or rifampicin is effective for hyperendemic trachoma. ${ }^{12}$ However, under field conditions the cost of delivery of such regimens of chemotherapy to the mass rural population is highly prohibitive. Alternatively, intermittent therapy with a topical tetracycline eye ointment twice daily for 5 to 7 days each month for a period of up to 6 months each year is recommended. ${ }^{3}$ Although this method of chemotherapy has been widely used, there is no evidence based on controlled studies to demonstrate its beneficial effects on preventing the blinding sequelae of trachoma by interfering with the transmission and reducing the severity of the disease.

Correspondence to Dr S. Darougar, Department of Clinical Ophthalmology, Institute of Ophthalmology, Judd Street, London WC1H 9QS.
Oral therapy with doxycycline is beneficial in the treatment of hyperendemic trachoma." In London treatment of patients suffering from paratrachoma (inclusion conjunctivitis and TRIC punctate keratoconjunctivitis) with a single dose of oral doxycycline $5 \mathrm{mg} / \mathrm{kg}$ body weight showed a marked clinical improvement for a period of up to 8 weeks and microbiologically a reduction of up to $50 \%$ in the Chlamydia trachomatis recovery rate (Darougar et al., in preparation).

In this report we present the result of a controlled, double-blind, stratified trial which was designed to assess the efficacy of family-based intermittent therapy of hyperendemic trachoma with oxytetracycline oily suspension in comparison with intermittent oral therapy with doxycycline in a village in Southern Iran. 


\section{Patients and methods}

SELECTION OF PATIENTS

The total population of 450 in Sar-Rig, a village in the Bandar-Abbas area of southern Iran, was examined for signs of active trachoma..$^{5}$ Patients with active trachoma in their whole conjunctiva were included in this study.

\section{CLINICAL EXAMINATION}

Eye examination was carried out with Haag-Streit slit-lamps. The signs observed in the whole conjunctiva, that is, upper tarsus and fornix and lower tarsus and fornix, were scored on a 0-3 scale and recorded separately for each area. The intensity of trachoma in the upper tarsus or the whole conjunctiva was calculated in accordance with the system described previously. ${ }^{2}$

\section{LABORATORY INVESTIGATION}

Conjunctival swabbings were collected from the whole conjunctiva of the right and left eyes. ${ }^{6}$ These were placed together in a plastic capsule containing 2SP transport medium ${ }^{7}$ with additional $3 \%$ fetal calf serum and stored in a liquid nitrogen refrigerator $\left(-180^{\circ} \mathrm{C}\right)$ until transported to the laboratory in London. Here the specimens were stored in a $-70^{\circ} \mathrm{C}$ refrigerator until the time of inoculation. The method of culture in irradiated McCoy cells and the identification of the isolates have been described elsewhere. ${ }^{8}$

\section{RANDOM ALLOCATION}

Patients with active trachoma were divided into 3 groups according to a randomisation schedule stratified for age, sex, intensity of trachoma, and the number of children with active trachoma in each family.

\section{MEDICATION}

Each group of patients was treated with one of the following 3 regimens of treatment. The first group received topical $1 \%$ oxytetracycline oily suspension twice daily for 7 days every month for 12 months. The second group received oral doxycycline $5 \mathrm{mg} / \mathrm{kg}$ body weight once a month for 12 months. The drug was given with a drink of milk to prevent vomiting. The control group received vitamin pills once a month for 12 months. All other members of the children's families, including parents, were also treated with the same regimen of treatment, but they were not included in this study. The treatment was given by 3 field technicians, each being responsible for one regimen of treatment.

FOLLOW-UP INVESTIGATIONS

After initial clinical and laboratory investigations treatment was started. The follow-up clinical examinations with the collection of conjunctival swabbings were made 4 months and 12 months after the onset of treatment.

\section{STATISTICS}

Chi-square significance tests were used for the comparison of results.

\section{Results}

Initially 147 patients with active trachoma in their whole conjunctiva were included in this trial. Of these, 18 were excluded because of inadequate treatment or follow-up. The allocation of the 129 remaining patients to the 3 treatment groups and their ages and sex distribution are shown in Table 1.

Examining the whole conjunctiva 4 months after the start of treatment, we found no marked difference in the cure rate or the number of patients with moderate to severe trachoma between the groups treated with topical oxytetracycline or doxycycline and the control group (Fig. 1). However, after 12 months of treatment the cure rates in the groups treated with oxytetracycline or doxycycline were $50 \%$ and $45 \%$ respectively compared with $28 \%$ in the control group (Table 2, Fig. 1). The difference between the results of treatment with the antibiotics compared with the control group reaches statistical significance at a level of $\mathbf{P}<0.05$. The difference between the efficacy of the 2 antibiotics was not statistically significant. There was also a marked reduction in the intensity of trachomatous inflammatory responses in the patients who were not cured. The percentage of patients with moderate to severe trachoma was reduced from $70 \%$ to $49 \%$ for the control group compared with a reduction from $84 \%$ to $21 \%$ for oxytetracycline and from $77 \%$ to $25 \%$ for doxycycline (Table 2, Fig. 1 ).

The changes in trachomatous inflammatory responses observed in the upper tarsal area alone in groups treated with topical oxytetracycline or oral doxycycline in comparison to the control groups are shown in Table 3 and Fig. 2. In general the response to treatment after 4 months and 12 months

Table 1 Allocation of patients to various treatments and their age and sex distribution

\begin{tabular}{llllllll}
\hline & \multicolumn{9}{l}{$\begin{array}{l}\text { Age } \\
\text { Treatment }\end{array}$} & patients & $0-1$ & $2-4$ & $5-9$ & $10-11$ & $\begin{array}{l}M / F \\
\text { (ratio) }\end{array}$ \\
\cline { 3 - 6 } & 47 & 2 & 21 & 17 & 7 & $20 / 27$ \\
Control & 38 & 4 & 15 & 10 & 9 & $12 / 26$ \\
Oxytetracycline & 44 & 4 & 11 & 21 & 8 & $20 / 24$ \\
\hline
\end{tabular}




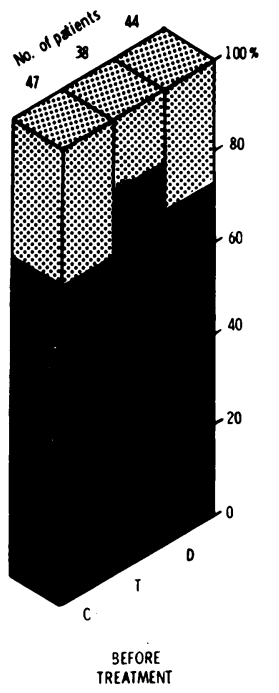

Moder ate or severe trachoma

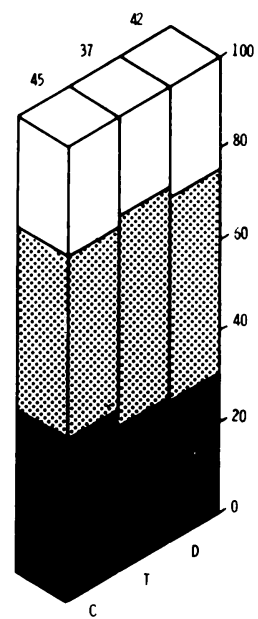

FOUR NIONTHS AFTER START OF AREATMENT
TRER STARTOF

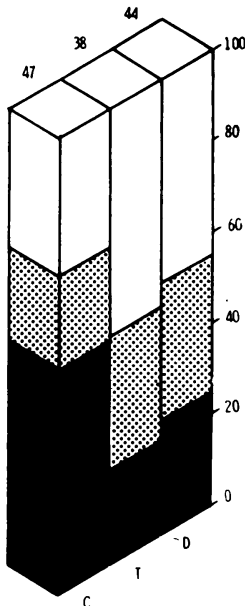

RUELVE MONV: $S$ AFTER START OF
Fig. 1 Changes in the grades of intensity of trachoma in the whole conjunctiva after intermittent therapy with topical oxytetracycline oily suspension $(T)$, oral doxycycline $(D)$, and placebo $(C)$.

Table 2 Changes in grades of intensity of trachomatous inflammatory responses in the whole conjunctiva following 12 months of intermittent treatment with topical oxytetracycline, oral doxycycline, or placebo

\begin{tabular}{|c|c|c|c|c|c|c|c|}
\hline \multirow{3}{*}{$\begin{array}{l}\text { Type of } \\
\text { treatment }\end{array}$} & \multirow{3}{*}{$\begin{array}{l}\text { No. of } \\
\text { patients }\end{array}$} & \multicolumn{6}{|c|}{ Number of patients with different grades of intensity* } \\
\hline & & \multicolumn{3}{|c|}{ Before treatment } & \multicolumn{3}{|c|}{ After treatment } \\
\hline & & $0-1$ & 2 & $3-4$ & $0-1$ & 2 & $3-4$ \\
\hline Placebo & 47 & 0 & $14(30 \%)$ & $33(70 \%)$ & $14(28 \%)$ & $10(23 \%)$ & $23(49 \%)$ \\
\hline Oxytetracycline & 38 & 0 & $6(16 \%)$ & $32(84 \%)$ & $19(50 \%)$ & $11(29 \%)$ & $8(21 \%)$ \\
\hline Doxycycline & 44 & 0 & $10(23 \%)$ & $34(77 \%)$ & $20(45 \%)$ & $13(30 \%)$ & $11(25 \%)$ \\
\hline
\end{tabular}

*0-1 = Inactive. $2=$ Mild. 3-4= Moderate to severe.

Table 3 Changes in grades of intensity of trachomatous inflammatory responses in the upper tarsal conjunctiva following 12 months of intermittent treatment with topical oxytetracycline, oral doxycycline, or placebo

\begin{tabular}{|c|c|c|c|c|c|c|c|}
\hline \multirow{3}{*}{$\begin{array}{l}\text { Type of } \\
\text { treatment }\end{array}$} & \multirow{3}{*}{$\begin{array}{l}\text { No. of } \\
\text { patients }\end{array}$} & \multicolumn{6}{|c|}{ Number of patients with different grades of intensity* } \\
\hline & & \multicolumn{3}{|c|}{ Before treatment } & \multicolumn{3}{|c|}{ After treatment } \\
\hline & & $\overline{0-1}$ & 2 & $3-4$ & $\overline{0-1}$ & 2 & $3-1$ \\
\hline Placebo & 47 & $2(4 \%)$ & $12(26 \%)$ & $33(70 \%)$ & $21(45 \%)$ & $8(17 \%)$ & $18(38 \%)$ \\
\hline Oxytetracycline & 38 & $2(5 \%)$ & $8(21 \%)$ & $28(74 \%)$ & $27(71 \%)$ & $4(11 \%)$ & $7(18 \%)$ \\
\hline Doxycycline & 44 & $2(5 \%)$ & $11(25 \%)$ & $31(70 \%)$ & $27(61 \%)$ & $8(19 \%)$ & $9(20 \%)$ \\
\hline
\end{tabular}

*0-1 = Inactive. 2 = Mild. 3-4= Moderate to severe. 
Fig. 2 Changes in the grades of intensity of trachoma in the upper tarsal conjunctiva after intermittent therapy with topical oxytetracycline oily suspension $(T)$, oral doxycycline $(D)$, and placebo $(C)$.
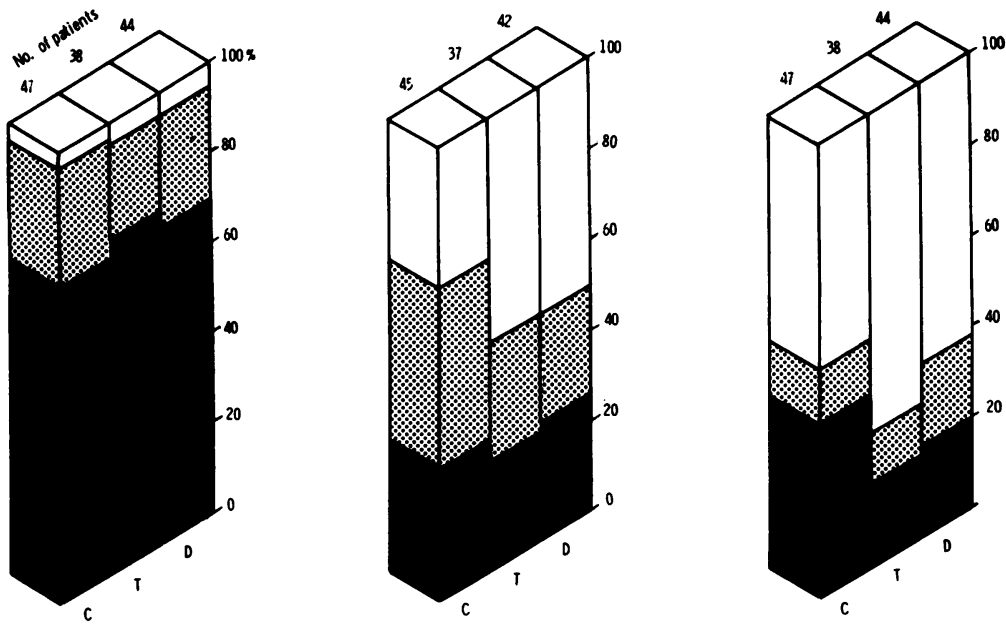

Moder ate or severe trachom

Mild trachoma with either of the antibiotics was more apparent in the upper tarsal conjunctiva than in the whole conjunctiva (Figs. 1 and 2).

Microbiologically $47 \%$ of patients allocated to the control group were positive for Chlamydia trachomatis before treatment compared with $26 \%$ and $36 \%$ of patients in the topical oxytetracycline or oral doxycycline groups respectively. Follow-up tests 4 months after the start of treatment showed a marked decrease in the positivity rate for $C$. trachomatis in the group treated with topical oxytetracycline (Table 4). After 12 months of treatment the positivity rate for $C$. trachomatis in the groups treated with oxytetracycline or doxycycline was $3 \%$ and $7 \%$ respectively compared with $19 \%$ in the control group. The difference in the rates of positivity for $C$. trachomatis between the treated groups and the control group is significant at a level of $\mathbf{P}<0.05$. The difference between the positivity rates following the treatment with the 2 antibiotics for 12 months was not statistically significant.

Table 4 Number of positive cultures for C. trachomatis before and after chemotherapy

\begin{tabular}{|c|c|c|c|c|}
\hline \multirow{3}{*}{$\begin{array}{l}\text { Type of } \\
\text { Treatment }\end{array}$} & \multirow{3}{*}{$\begin{array}{l}\text { No. of } \\
\text { patients }\end{array}$} & \multicolumn{3}{|c|}{ No. of positive cultures } \\
\hline & & \multirow{2}{*}{$\begin{array}{l}\text { Before } \\
\text { treatment }\end{array}$} & \multicolumn{2}{|c|}{ After treatment } \\
\hline & & & 4 months & 12 months \\
\hline Placebo & 47 & $22(47 \%)$ & $7(16 \%)$ & $9(19 \%)$ \\
\hline Oxytetracycline & 38 & $10(26 \%)$ & $1(3 \%)$ & $1(3 \%)$ \\
\hline Doxycycline & 44 & $16(36 \%)$ & $7(17 \%)$ & $3(7 \%)$ \\
\hline
\end{tabular}

\section{Discussion}

In this trial preschool and school children with active trachoma were included. Stratified randomisation for age, sex, and grades of intensity of trachomatous inflammatory responses produced a well balanced distribution of patients in the groups allocated to the active and placebo treatments (Tables 1 and 2, Figs. 1 and 2). Microbiologically, before treatment the number of patients positive for $C$. trachomatis was lower in the group allocated to the topical oxytetracycline than in the other 2 groups (Table 4).

During the follow-up studies of the control group we found a considerable degree of natural decline in the prevalence of trachoma, in the intensity of inflammatory responses, and in the positivity rate for $C$. trachomatis (Tables 2, 3, and 4, Figs. 1 and 2). The degree of natural decline of trachoma in our control group was much higher than that observed in Tunisia. ${ }^{1}$ Apart from the effect of increasing age this could be attributed in part to the reduction in the level of interfamily transmission in the village following therapy with antibiotics of the majority of the cases of active trachoma (reservoirs of infection) included in the other 2 groups and to the steady improvement of socioeconomic conditions in the village.

This study showed that a 4-month course of intermittent therapy with either topical oxytetracycline or oral doxycycline is not sufficient to reduce the intensity of trachomatous inflammatory responses in the conjunctiva. But when the treatment 
was continued for 12 months a marked decrease in the prevalence of trachoma and in the grades of intensity of inflammatory responses as well as the positivity rate for $C$. trachomatis was observed (Figs. 1 and 2).

Assessing the inflammatory changes of trachoma in the upper tarsal conjunctiva alone, we found the cure rate for all 3 groups was approximately $30 \%$ higher than that obtained by examining the whole conjunctiva (Tables 2 and 3, Figs. 1 and 2). This finding confirms our previous observation that active but mild to moderate trachomatous inflammatory changes may persist in the upper fornix and the lower lid conjunctivae of some of the patients with cured upper tarsal conjunctiva. This residual inflammation in the upper fornix and the lower lid is shown to be related to the increased risk of recurrence of moderate and severe trachoma in the whole conjunctiva, including the upper tarsal area. ${ }^{2}$

In assessing the result of this trial we may conclude that intermittent therapy with topical oxytetracycline or oral doxycycline are both effective against trachoma clinically and microbiologically if they are used for a period of 1 year. While no marked difference was observed between the efficacy of these 2 regimens of mass chemotherapy, the monthly intermittent therapy with a single dose of doxycycline offers the advantage of being more practical and less expensive for mass control of trachoma by requiring approximately one-tenth of the staff, transport, and other facilities needed for the intermittent topical therapy with tetracycline eye ointment.
The authors are grateful to the Dean of the School of Public Health, Teheran, and the Director of the Department of Health and Welfare, Bandar-Abbas, for their support of this project.

We are indebted to Professor $\mathbf{H}$. Mohsenine for administrative duty and to $\mathrm{Mr} \mathrm{H}$. Shah-Mohamadi for his assistance in the collection of clinical specimens.

The research project was partially supported by grants from the Dulverton Trust, the Wellcome Foundation, the Order of St John, and an anonymous donor.

\section{References}

${ }^{1}$ Dawson CR, Hoshiwara I, Daghfous T, Messadi $M$, Vastine DW, Schachter J. Topical tetracycline and rifampicin therapy of endemic trachoma in Tunisia. Am J Ophthalmol 1975; 79: 803-811.

${ }^{2}$ Darougar S, Jones BR, Viswalingam $\mathrm{N}$, et al. Topical therapy of hyperendemic trachoma using rifampicin, oxytetracycline or spiramycin eye ointments. Br J Ophthal$\mathrm{mol}$ in press.

${ }^{3}$ Tarizzo M, ed. Field Methods for the Control of Trachoma. Geneva: World Health Organisation 1973; 9.

${ }^{4}$ Hoshiwara I, Ostler HB, Hanna L, Cignetti F, Coleman VR, Jawetz E. Doxycycline treatment of chronic trachoma. JAMA 1973; 224: 220-223.

${ }^{5}$ Dawson CR, Jones BR, Darougar S. Blinding and nonblinding trachoma: assessment of intensity of upper tarsal inflammatory disease and disabling lesions. Bull WHO 1975; 52: 279-282.

'Darougar S, Jones BR. Conjunctival swabbing for the isolation of 'TRIC' agent (Chlamydia). Br J Ophthalmol 1971 55: $585-590$.

'Gordon FB, Harper IA, Quan AL, Treharne JD, Dwyer RStC, Garland JA. Detection of Chlamydia (Bedsonia) in certain infections in man. 1. Laboratory procedures: comparison of yolk sac and cell culture for detection and isolation. J Infect Dis 1969; 120: 451-462.

${ }^{8}$ Darougar S, Kinnison JR, Jones BR. Simplified irradiated McCoy cell culture for isolation of Chlamydiae. In: Nichols RL, ed. Trachoma and Related Disorders caused by Chlamydial Agents. Amsterdam: Excerpta Medica, 1970; 63-70. 